\title{
El plan S para acelerar el acceso abierto: contexto, retos y debate generado
}

\author{
ERnest Abadal \\ Universitat de Barcelona \\ abadal@ub.edu
}

https://orcid.org/0000-0002-9151-6437

\author{
Alexandre López-Borrull \\ Universitat Oberta de Catalunya \\ alopezbo@uoc.edu
}

https://orcid.org/0000-0003-1609-2088

\author{
Candela Ollé-Castellà \\ Universitat Oberta de Catalunya \\ candela.olle@gmail.com \\ https://orcid.org/0000-0002-8302-4790
}

Francesc García-Grimau

Universitat de Barcelona

francesc.garcia.grimau@gmail.com

https://orcid.org/0000-0001-8969-7868

\section{Plan S to accelerate open access: context, challenges and generated debate}

\section{RESUMEN ABSTRACT}

Se analizan los principales elementos y características del Plan S, iniciativa de la cOAlition S, promovido desde Europa para acelerar el acceso abierto, al pedir a los investigadores que los artículos sufragados por un conjunto de agencias adheridas sean puestos a disposición inmediatamente una vez publicados, con una serie de requisitos a nivel de licencias. Dicho plan ha conllevado un encendido debate en el mundo académico entre defensores y detractores. $A$

continuación, se sintetizan y categorizan los principales argumentos y puntos de vista que se han manifestado contrarios a la iniciativa. Finalmente, se apuntan los principales retos que el Plan $\mathrm{S}$ tendrá para cumplir con sus objetivos.

PALABRAS CLAVE

Plan S, Revistas científicas, Repositorios; Acceso abierto; Ciencia abierta; cOAlition S
The main elements and characteristics of Plan S, initiative of cOAlition S, promoted from Europe to accelerate open access, by asking researchers that articles paid by a set of adhered agencies to be made available immediately once published, with a series of licensing requirements. This plan has led to a heated debate in the academic world between defenders and detractors. The main arguments and points of view that have been contrary to the initiative are summarized and categorized below. Finally, the main challenges that Plan S will have to meet its objectives are noted.

KEYWORDS

Plan S; Scientific journals; Repositories; Open access;

Open science; cOAlition $S$ 


\section{Introducción}

El Plan S (Science Europe, 2018, 2019), una iniciativa de cOAlition S, del Consejo Europeo de Investigación (ERC) y de varias agencias estatales europeas, ha conseguido un destacado protagonismo en el ámbito de la investigación y de la comunicación científica desde que se hizo pública su primera versión, el 4 de septiembre de 2018 y se ha mantenido también con posterioridad a su actualización el 31 de mayo de 2019. Es una propuesta que quiere acelerar la transición hacia el acceso abierto (y esto queda bien claro en el subtítulo de la primera versión: "Accelerating the transition to full and immediate Open Access to scientific publications") y que tiene por objetivo conseguir que, a partir de 2021, todas las publicaciones científicas derivadas de proyectos con financiación pública se publiquen en acceso abierto de manera inmediata.

Sus principales promotores y valedores han sido Robert-Jan Smits, Open Access Envoy de la Comisión Europea, y Marc Schiltz (2018), presidente de Science Europe así como JeanClaude Burgelman, promotor de la ciencia abierta en Europa desde la Dirección General de Investigación. El plan cuenta con el apoyo de la Comisión Europea y del ERC y eso le ha dado un empuje y un reconocimiento de muy alto nivel. Como se puede ver en su portal web, dispone también del apoyo de asociaciones de universidades (LERU, EUA), de sociedades académicas, y de organizaciones relacionadas con el acceso abierto (como COAR, SPARC, LIBER).

Los antecedentes del plan se encuentran en la declaración de Amsterdam (2016) sobre la ciencia abierta, un documento aprobado por la Unión Europea durante la presidencia semestral de los Países Bajos en el que se pedía que todas las publicaciones científicas derivadas de proyectos con financiación pública estuvieran en acceso abierto a partir de 2020 (por eso, la primera versión del plan S incluía esta fecha como meta). La poca concreción sobre cómo conseguir este objetivo llevó a la presidencia de la Comisión Europea a hacer un encargo a R-J Smits para sacar adelante un plan que permitiera acelerar la transición hacia el acceso abierto. La motivación de la Comisión Europea es clara: aporta muchos millones para la financiación de la investigación y quiere que los resultados estén disponibles de inmediato para toda la ciudadanía. Siguiendo esta línea argumental, además, es importante resaltar que el plan basa su estrategia en la alianza con las agencias financiadoras de la investigación, que sirven para asegurar la máxima eficacia en los objetivos propuestos.

De momento, se han adherido al Plan $S$ las principales agencias públicas de financiación de Austria, Finlandia, Francia, Irlanda, Luxemburgo, Países Bajos, Reino Unido, Eslovenia, Polonia y Noruega, así como alguna agencia de Italia y Suecia (un total de 16 agencias de 14 países, dos de los cuales, Jordania y Zambia, no son europeos), las agencias privadas Wellcome y la Bill \& Melinda Gates Foundation, así como las organizaciones internacionales World Health Organization y TDR (el programa especial de Training in Tropical Diseases). El European Research Council (ERC), como se ha comentado anteriormente, también respalda el Plan $\mathrm{S}$ y está trabajando en su implementación.

Se han realizado algunos cálculos sobre la producción científica que está afectada por dicha financiación. Según el estudio llevado a cabo por la empresa Delta en la base de datos Dimensions, estaríamos hablando que un 3,3\% de la producción mundial (Pollock y Michael, 2018) está financiada por alguna de las agencias adheridas al plan S. Por su parte, un informe del Institute for Scientific Information (ISI) los sitúa en el 6,4\% de las publicaciones en WoS en 2017 (Quaderi, 2019), con un total de 120.000 artículos publicados en 10.000 revistas.

El plan $\mathrm{S}$ ha generado mucho debate en el ámbito académico y también en el editorial y, curiosamente, ha concitado críticas tanto de sectores contrarios como favorables al acceso abierto. Además de estos textos, que se han difundido en revistas y blogs, la propia cOAlition 5 (2019) publicó los más de 600 comentarios recibidos, tanto de particulares como de organizaciones en la consulta pública que se mantuvo abierta hasta el 9 de febrero y que originó la segunda versión del documento, que ha retocado varios aspectos del plan (una transición un poco más larga, clarificación del papel de los repositorios, p.e.) y que, en general, ha sido bien recibida por todas las partes.

Las principales organizaciones que han manifestado sus opiniones son los editores de revistas de suscripción (SAGE, Springer, Taylor and Francis, Wiley, en especial), editores de revistas de acceso abierto (Hindawi, entre otros), editores de sociedades científicas, asociaciones de editores (ALPSP, STM), academias científicas (European Federation of Academies of Sciences and Humanities, The Global Young Academy, All European Academies), universidades (University College London, University of Oulu, Imperial College, Univ. Southampton, Cambridge University, etc.), asociaciones de universidades (LERU, EUA), asociaciones de bibliotecas (LIBER) y entidades vinculadas al movimiento de acceso abierto (COAR, SPARC Europe, OpenAIRE). Y también, evidentemente, han hecho oír sus voces los propios científicos, con varias cartas abiertas (Kamerlin et al, 2018; Eisen, 2018; Willighagen y Tennant, 2019) así como varios comentaristas del ámbito de la comunicación científica (Schneider, 2018, 2019; Kingsley, 2019; Poynder, 2019a). El punto de vista español puede encontrarse en diversas referencias (Martínez-Galindo et al, 2019, Sánchez-García, 2019).

Aunque la cOAlition S se centra en el contexto europeo, la incidencia que tiene el plan en el sistema global de comunicación científica ha hecho que también se hayan posicionado países como la China, o que se haya publicado otra propuesta con la visión de América Latina, AmeliCA, que tiene el mismo objetivo (el acceso abierto) pero con una estrategia distinta, que ya se 
comentará más adelante (Becerril-García, 2019).

En cualquier caso, está claro que se ha hablado mucho del plan S, aunque, en general, se tiene un desconocimiento tanto de los detalles como de los argumentos en contra que se han dado a conocer. Por ello, con este texto, queremos ofrecer un análisis sobre el debate generado, que contribuya a tener una visión panorámica y sistemática de la cuestión.

\section{Objetivos y metodología}

El objetivo de nuestro estudio consiste en analizar y valorar las características fundamentales del Plan 5 y también sintetizar los principales argumentos y puntos de vista que se han manifestado contrarios a la iniciativa.

De manera más precisa, queremos:

\section{- Sistematizar los argumentos en contra}

- Determinar los principales grupos reticentes

- Valorar globalmente la propuesta

Nuestro estudio se basa en un análisis de contenido de las dos versiones publicadas del plan S, así como de los principales documentos publicados que las han comentado, ya sea en artículos de tipo científico, informes de posicionamiento de organizaciones y empresas involucradas (que podemos considerar stakeholders del Plan S), así como en artículos en blogs y redes sociales. Para ello se ha usado principalmente el término de búsqueda "Plan S" durante el período que va desde septiembre 2018 hasta julio del 2019. En el caso de Scopus, por ejemplo, se encuentran disponibles 65 documentos con este término en el título, resumen o palabras clave, la mayoría de los cuales, no obstante, recogen posicionamientos de los diversos agentes implicados, así como algunas réplicas.

De los comentarios publicados se han extraído y sistematizado los principales argumentos, que se comentan y se contextualizan con mayor detalle. Es importante tener en cuenta que nuestro estudio pone el foco en las reacciones contrarias al plan pero que esto no tiene ninguna relación con la intensidad del rechazo o de la adhesión que suscita el plan S. No se indican los posicionamientos favorables, que han sido muchos.

En la sección 3 se sistematizan y contextualizan las principales características del plan 5 y en la sección 4 se sintetizan las críticas principales que se le han hecho, intentando mostrar también el perfil de las organizaciones que se han manifestado en contra.

\section{Elementos y características del Plan S}

En este apartado vamos a sintetizar y comentar los principios fundamentales del plan S, según su última actualización, aunque en algunos casos nos vamos a referir a su vez a los cambios que se han suscitado respecto a la versión inicial. El análisis se basa en el documento "Principles and implementation", que consta tres partes: un decálogo de principios básicos ("The Plan 5 Principles"), una guía de implementación y los requerimientos técnicos ("Technical Guidance and Requirements").

El principio fundamental es que todas las publicaciones (derivadas de proyectos financiados por las agencias suscriptoras del plan) deben estar en acceso abierto de manera inmediata.

"With effect from 2021, all scholarly publications on the results from research funded by public or private grants provided by national, regional and international research councils and funding bodies, must be published in Open Access Journals, on Open Access Platforms, or made immediately available through Open Access Repositories without embargo."

Teniendo este objetivo como visión general, vamos a comentar los principales detalles y especificaciones que se indican en el documento fundacional del plan S.

\subsection{Plazo y alcance}

Aunque en la primera versión se indicaba el año 2020, el plazo se ha extendido hasta 2021 para dar un poco más de tiempo a la preparación de toda la operativa. Así pues, la obligación de publicar en acceso abierto es para todas las publicaciones derivadas de convocatorias que se hagan públicas a partir del 1 de enero de 2021.

En cuanto al alcance, el Plan $\mathrm{S}$ se centra en los artículos científicos, dejando para un futuro inconcreto otros tipos de documento como las monografías o los capítulos de libro (principio adicional 7). En la primera versión, se comentaba que una vez iniciado el Plan 5 en lo que respecta a los artículos ya se actuaría a continuación sobre los otros formatos de difusión científica. Cabe tener en cuenta que las monografías y los capítulos de libro son un tipo de documento con un grado de apertura más bajo y van a requerir otro tipo de estrategia. La cOAlition $\mathrm{S}$ se compromete a definir un plan específico para las monografías a finales de 2021.

\subsection{Licencias}

El primer principio indica claramente que las publicaciones deben usar licencias CC-BY, la tipología más amplia y sin exclusividad, ya que permiten no tan sólo la libre reproducción y distribución de los contenidos, sino que también posibilitan la reutilización (traducciones y adaptaciones, especialmente) y la posible comercialización (ya sea mediante la venta de versiones impresas o en otras formas). De esta forma se garantiza 
un libre acceso total a los contenidos. Se trata de un tipo de licencia que hasta ahora ha tenido un papel minoritario en la difusión de contenidos académicos. También se demanda que los autores conserven los derechos.

\subsection{Modelo de negocio de las revistas}

En lo referente al posicionamiento respecto los modelos de negocio de las revistas científicas, se deben destacar dos cuestiones: los fondos asignados se pueden usar para pagar los APC (Article Processing Charges, las tasas que los autores pagan por publicar) y explícitamente, el Plan $\mathrm{S}$ se posiciona en contra de las revistas híbridas. Además, se indica que las tasas de publicación que se sufragarán serán "razonables". En este sentido se supone que deben seguir iniciativas como Fair Open Access Alliance (FOOA, 2019) que piden a las revistas unos APC transparentes y proporcionados al trabajo editorial realizado. Por lo que se refiere al modelo híbrido, mientras que en la primera versión del plan incluía una prohibición categórica para utilizar este tipo de revista, actualmente podría publicarse en ellas, pero no se podrían utilizar fondos de la financiación pública para pagar sus APC.

Asimismo, se incorporó el reconocimiento de las revistas en acceso abierto que no exigen el pago de APC, que son ciertamente relevantes en ciencias sociales, así como las editoriales universitarias.

A los modelos y la financiación de costes editoriales se refieren los principios adicionales 4, 5 y 8, algunos de los cuales como se verá en el apartado 4, han sido los que han generado mayor número de comentarios y críticas.

\subsection{Vías de cumplimiento y requerimientos del Plan S}

Para el cumplimiento por parte de los investigadores, el Plan S propone tres vías:

a) La publicación del artículo en revistas o plataformas de acceso abierto

En este caso, las revistas deben cumplir un conjunto de requisitos obligatorios y algunos otros de opcionales.

b) El depósito en repositorios (para artículos en revistas de suscripción)

Se podrá publicar en revistas de suscripción que permitan depositar la versión publicada (Version of Record, VoR) o la versión revisada (Author's Accepted Manuscript, AAM). Recordemos que debe ser de manera "inmediata", sin periodos de embargo. Se pretende acabar con una visión de preprint de contenido diferente al publicado en la revista. La consideración de la vía verde (los repositorios) constituye un cambio notable respecto de la primera versión de plan y es un indicativo de su importancia para difundir y preservar los resultados de la investigación. (El grupo editorial Lancet, por ejemplo, ya ha aceptado estos requerimientos y permite archivar en un repositorio la versión revisada sin ningún coste y con licencia CC BY).

\section{c) Revistas de suscripción en transición}

Constituye una tercera vía, pensada para revistas que dispongan de un plan de transición al modelo de acceso abierto. Se podrá publicar en ellas y tener financiación para ello hasta 2024.

A partir de las tres vías descritas para cumplir con el plan S, Bosman y Kramer (2019) han descrito hasta nueve rutas potenciales que permite el Plan S (cuatro doradas, tres verdes y dos híbridas).

\subsection{Requerimientos técnicos}

La parte III del documento del plan S se centra en la descripción detallada de una amplia lista de requisitos técnicos (pero también legales y de modelo de negocio) que deben cumplir las revistas, plataformas y repositorios para ajustarse al modelo establecido (indicado también en el principio adicional 2).

Estos requerimientos pretenden favorecer la creación de un ecosistema de revistas y de plataformas de acceso abierto de calidad. Para ello las revistas deben estar en el directorio DOAJ, deben ser transparentes en lo referente a las políticas editoriales de calidad, cumplir con los aspectos éticos, coste y posibles descuentos en función de los países de los investigadores, usar identificadores persistentes como ORCID, tener políticas de preservación digital, políticas de compartición de datos de investigación y estándares de citas abiertas, entre otros elementos. Para muchas revistas, sobre todo en el caso de las ciencias sociales y las editoriales universitarias pequeñas, el cumplimiento de todos ellos representa un reto notable (López-Borrull et al, 2019).

\subsection{Criterios de evaluación}

Finalmente, los financiadores acuerdan que en el proceso de evaluación de la investigación se valorará el mérito intrínseco del trabajo y no el canal de publicación, el factor de impacto o la editorial (principio adicional 10). Esta consideración, muy importante para avanzar en el despliegue de la ciencia abierta, se incorporó en la segunda versión del plan debido a los comentarios recibidos. Así pues, se reconoce que debe considerar el valor del artículo y no el de la revista, siguiendo las recomendaciones de la San Francisco Declaration on Research Assessment DORA (2012) y del manifiesto de Leiden (Hicks et al, 2015).

Se debe señalar asimismo que el Plan S es un paso más dentro de la apuesta de las instituciones por la ciencia abierta --en 
la visión descrita por Anglada y Abadal (2018)-- y que incorpora aspectos fundamentalmente relacionados con el acceso abierto, pero también con los datos FAIR y con una evaluación de los contenidos que no dependa de las Journal Level Metrics (JLM).

\section{Argumentos en contra}

A continuación, se describe y sistematiza el impacto que el plan $\mathrm{S}$ ha tenido sobre la comunidad académica, así como las principales críticas que ha recibido, tanto por parte de defensores del acceso abierto como por detractores de los principios que emanan de él. Diversos textos han resumido algunas de las críticas (Quaderi, 2019; Bernal, 2018, López-Borrull, 2019) y también se encuentran páginas web que disponen de listas con las repercusiones y opiniones (Kingsley, 2019).

Cabe destacar, como se ha descrito en la introducción, el gran número de actores y grupos de interés que se han posicionado en relación al Plan S. Así, respecto a algunos de los aspectos más críticos en los últimos años, como el modelo de negocio de las revistas, se han posicionado desde defensores de las revistas híbridas (recordemos, el principal actor contra el que se posiciona el plan S), hasta críticos con el hecho que se mantengan las APC. En este segundo caso, buena parte provienen del propio movimiento de acceso abierto.

Hemos agrupado las principales críticas en cuatro grandes ámbitos: cuestiones generales (libertad académica, licencias, evaluación, etc.), críticas al modelo APC, dudas sobre la apuesta por la vía verde y, finalmente, la dificultad de su encaje en un sistema de comunicación científica globalizado.

\subsection{Cuestionamiento general del Plan S}

Algunas de las cuestiones generales de fondo con las que los académicos y algunas sociedades científicas se han posicionado respecto el Plan $\mathrm{S}$ se describen a continuación:

\subsubsection{Constituye una limitación de la libertad académica del investigador}

En una carta abierta, Reaction of Researchers to Plan S: Too Far, Too Risky (Kamerlin et al, 2018), suscrita por más de 1700 científicos hasta el momento (con un porcentaje elevado de investigadores de los países cuyas agencias financiadoras han respaldado el Plan S), se llevan a cabo diversas críticas, entre las cuales el hecho de que se atenta contra la "libertad académica", ya que no deja publicar a los investigadores donde quieran hacerlo. Así, el parámetro del cumplimiento de los requisitos del Plan $\mathrm{S}$ pasa en algunas disciplinas a limitar de forma evidente el rango de revistas donde los investigadores pueden publicar.

Por poner un ejemplo, en la categoría temática de Chemistry (Miscellanous) que recoge el Scimago Journal \& Country Rank (SJR), de las 449 revistas recogidas, tan sólo 65 son revistas en acceso abierto, y ello como hemos indicado no quiere decir que cumplan con el conjunto de requisitos que el Plan $\mathrm{S}$ pide a las revistas. Más que una contraargumentación, puede debatirse el hecho de que actualmente y debido a los procesos de evaluación existe un vector que es la elección por factor de impacto. Si se añade un segundo eje que sería el del cumplimiento del Plan S, en algunas disciplinas puede pasar que la elección de las revistas puede llegar a ser un auténtico quebradero de cabeza. Aun así, es más que probable que la tercera vía de publicación (revistas en acuerdo transformativo) pueda permitir una transición menos traumática en algunas áreas temáticas que en otras.

\subsubsection{La licencia preferida es demasiado abierta}

Según algunos detractores, la obligación de usar las licencias CC-BY, que permiten la comercialización y la reutilización de los contenidos puede generar problemas a algunos autores porque se limita su libertad de restringir los usos comerciales.

Cabe destacar que, de hecho, este debate, igual que en el caso de los datos de investigación se relaciona con la reutilización de contenidos y con su posible valorización, elementos hasta ahora poco explorados. Así, por ejemplo, en el blog de divulgación científica de Francis Villatoro (2019) ya se expone el posible giro que la minería de artículos científicos puede dar a la investigación. La reutilización de contenidos mediante la minería de datos, herramientas de big data y la inteligencia artificial pueden dar un nuevo valor a aquello ya publicado. Para que se genere una industria potente y alternativa a las editoriales tradicionales con muchos años de producción científica con sus derechos de autor por explotar, es necesario que se puedan abrir e incluso permitir la nueva visión comercial con ellos. No sería, pues, una cuestión de nuevos compendios, sino de generar valor en datos masivos.

\subsubsection{Puede tener efectos no aclarados sobre evaluaciones de los académicos}

Hasta ahora el modelo de evaluación de la publicación se basa en los índices de impacto. El Plan S debería funcionar sobre otros parámetros que no están todavía implementados. La aplicación de los principios del Plan S, por lo tanto, puede afectar negativamente, a corto plazo, a la promoción de jóvenes investigadores. Sobre todo, en un período en el que puedan coexistir dos sistemas alternativos de evaluación.

La implementación de una nueva forma de evaluar, basada en el contenido y el valor intrínseco del artículo exige unos recursos y unos cambios de mentalidad que no serán fáciles. El propio plan $\mathrm{S}$ lo ha reconocido explícitamente en la segunda 
versión, tal y como se indicaba anteriormente. Aun así, este hecho siempre va a existir y lo que debe llevarse a cabo es una transición ordenada y razonable, de la misma forma que las instituciones llevan a cabo cuando generan una política propia de acceso abierto o de respaldo a la Declaración de San Francisco (DORA, 2012) o al manifiesto de Leiden (Hicks et al, 2015).

La evolución tecnológica y las críticas sistemáticas, así como la apuesta por la ciencia abierta están llevando el modelo actual de evaluación a una crisis de confianza y de continuidad. Pequeños giros en el relato científico alternativo al factor de impacto como pretende o facilita el Plan $\mathbf{S}$ pueden ser los pequeños cambios necesarios para ayudar a aquellas instituciones que pretendan iniciar nuevos mecanismos de evaluación de la investigación.

\subsection{Críticas a los APC como modelo de negocio de las revistas científicas}

Como se ha comentado anteriormente, uno de los aspectos principales del Plan 5 es que intenta equilibrar el ecosistema de comunicación científica por la vía de intentar eliminar los modelos híbridos en las revistas científicas, que fueron la vía escogida por las revistas de suscripción para permitir que los investigadores publicaran en ellas y, a la vez, dieran acceso abierto. De alguna forma, las principales editoriales permitían parcialmente el acceso abierto en las revistas con mayores factores de impacto, las preferidas para proyectos competitivos. El Plan 5 pretende acabar con esta doble vía de ingresos al coexistir el pago por suscripción con el de publicación. Aun así y de la forma establecida en los principios, algunas de las críticas recibidas se basan en que:

\subsubsection{Se potencia el modelo editorial basado en el pago de} APC

Los principios y valores de AmeliCA (2019), un proyecto impulsado por Redalyc, CLACSO y otras organizaciones latinoamericanas, constituye un contundente alegato contra el pago de APC en las revistas y la defensa del modelo latinoamericano de financiación pública para las revistas, tal y como ha sido comentado y argumentado por Becerril (2019).

Sobre esta cuestión, además, Debat y Babini (2019) piensan que facilitar el coste de los APC desde las mismas entidades financiadoras, como hace el plan S, va a propiciar que el pago de tasas por publicar sea la vía preferente de negocio de las revistas científicas de las principales editoriales. Dentro del activismo por el acceso abierto, encontramos una parte de los científicos y actores que tendrían suficiente con el fin del modelo híbrido, pero igualmente existe otra parte que considera que el APC pasa a ser un peaje que no todo el mundo puede pagar para difundir sus resultados de investigación.

\subsubsection{Los autores no tienen las mismas oportunidades}

Otro argumento contra los APC se basa en que no ofrece igualdad de oportunidades a todos los autores. En este caso, las voces críticas provienen de las revistas de suscripción que argumentan que ellas no discriminan los autores que no pueden sufragar las tasas de publicación, modelo al que según ellos empuja el plan S. Esta cuestión podría conllevar problemas para las capas más débiles y menos competitivas dentro de la ciencia, como por ejemplo los jóvenes investigadores, jubilados, países con pocos recursos, autores sin financiaciones, entre otros (Kamerlin, 2018; Burgman, 2019).

A pesar de que el plan S demanda establecer costos "aceptables y transparentes" y que se realicen descuentos para países y organizaciones menos favorecidas, Debat y Babini (2019) dudan que sean adoptados por las editoriales científicas con lo cual se mantendrían, en su opinión, las barreras para muchos autores.

\subsubsection{El modelo APC puede tener sus propias perversiones}

Tal como explican de Bruin, Kamerlin et al (2019) el sistema de pago por publicar puede tener dos perversiones: publicar muchos artículos a precios reducidos o publicar muy pocos a precios muy altos (para así mantener el factor de impacto). Ambas perversiones afectarían directamente el sistema de revisión por expertos ya que no estaría condicionado por la calidad y el interés de los artículos sino por la posibilidad de disponer de mayores ingresos. Por este motivo, estos autores consideran que únicamente la ruta diamante/platino de acceso abierto (las revistas que no cobran tasas por publicar) daría sentido a los auténticos objetivos del movimiento del acceso abierto ya que posibilita las mismas condiciones de publicación para todos los autores.

\subsection{Dudas sobre el papel de la vía verde del acceso abierto}

El Plan S, tal y como se ha descrito, muestra que el investigador puede cumplir con los principios y obligaciones de las entidades de la cOAlition $\mathrm{S}$ optando por revistas de acceso abierto que cumplan con sus requisitos (o las que estén en un proceso de transformación). Ahora bien, también puede optar por el archivo en un repositorio, sin ningún tipo de embargo temporal, de la versión publicada (VoR) o de la versión aceptada (AAM) por la revista siempre y cuando el repositorio cumpla con los requisitos del Plan S.

En este caso, los principales aspectos que se han considerado en las críticas al plan $\mathrm{S}$ han sido: 


\subsubsection{Las condiciones de autoarchivo son exigentes para las revistas}

La conjunción de exigencias del plan $\mathbf{S}$ de que los autores retengan los derechos de explotación, de que la licencia sea la más abierta posible y que la versión de autoarchivo tenga el mismo contenido (aunque no formato) que el publicado hace difícil que las revistas permitan todos estos requerimientos por cuanto perjudica sensiblemente el modelo de pago por suscripción. Estas revistas, por tanto, se verían forzadas a optar por el pago de APC, como única alternativa económica para seguir publicando.

\subsubsection{Los requerimientos para los repositorios son exigen-} tes

Aunque los requerimientos técnicos obligatorios para los repositorios son menores que para las revistas hay que tener en cuenta, en este caso, que se exige la máxima interoperabilidad y además que la versión archivada ya no sea el preprint sino la versión publicada o aceptada. Ello puede suponer un desafío para los repositorios por cuanto va a conllevar el depósito de distintos tipos de archivos (anteriores al Plan 5 y posteriores), pero también la necesidad legal de controlar si los autores disponen o no de los derechos de autor para depositar la versión final. Cabe recordar los problemas legales que han tenido redes sociales académicas como ResearchGate en este sentido.

\subsection{Encaje geopolítico en un sector globalizado}

El hecho de que el Plan S se presente como la solución paneuropea (aunque no incluya de momento a Alemania o España, por ejemplo) explica que, en una perspectiva estratégica, debamos considerar los siguientes aspectos o críticas al Plan S:

\subsubsection{Es una propuesta parcial que pretende cambios globa- les}

La inexistencia de una política global sobre el acceso abierto puede ser un problema notable para las editoriales. Las condiciones de los mandatos son diferentes según los países y los financiadores, pero el alcance de las editoriales es global. Así, las editoriales (en especial, las sociedades científicas) operan en mercados globales y deben pensar en todos los autores (los que quieren pagar APC, pero también en los que no quieren pagar, los que ya les va bien colgar en repositorios con un periodo de embargo, etc.), cada uno de los cuales puede tener requerimientos diferenciados. Para un modelo de negocio que pudiera ser adaptable a todo ello, el modelo híbrido era una buena solución.

En este sentido, algunas editoriales señalan que la cOAlition $S$ debería ser consciente de que tiene una presencia minoritaria en la investigación y la publicación científica. Los cambios que pretende desencadenar (que las revistas se pasen directamente al modelo OA) chocan con la realidad de un mercado editorial globalizado en el que los editores deben tener respuesta para todas las situaciones. Así, fue muy bien recibido el apoyo de la China al Plan S. En este sentido, aunque no ha explicitado si hará suyos los principios del plan, sí que se ha manifestado mediante una petición a las editoriales científicas para que no se incrementen los precios, debido a los posibles sobrecostes

\subsubsection{Puede generar velocidades y comunidades distintas}

En la carta abierta suscrita por 1700 investigadores, se afirma que "podría crearse una dicotomía en la sociedad científica global, entre los países que respaldan el Plan 5 y el resto del mundo". La ciencia es global y los científicos se mueven libremente entre países y colaboran globalmente. Puede ser compleja la gestión de revistas vedadas para determinados investigadores por no cumplir los requisitos del plan $\mathrm{S}$ a la vez que editoriales que no tengan suscripciones en determinados países. La visión entre editoriales internacionales y estados con sus propios intereses de investigación y comunicación científica continúan chocando en el ecosistema actual, con el riesgo que la ciencia pueda ser una víctima colateral de dichas pugnas.

El Plan S pone precisamente en evidencia la ausencia de una estrategia global por parte de instituciones o grupos de interés, de forma que las soluciones se presentan a menudo como necesarias, pero no suficientes. Incluso, cabe ver cómo las estrategias propuestas desde Estados Unidos, Europa, China o el Global South no implican impedimentos mutuos y permiten que cada uno de ellos, a su ritmo, lleguen al mismo objetivo.

Actores globales como las fundaciones Wellcome y Gates apoyan la propuesta, dejando claro incluso que ellos hubieran ido más allá, como ya hacen en la financiación que gestionan para proyectos de investigación. En una editorial de la revista Conservation Biology (Burgman, 2019), crítica con el Plan S, se habla incluso de que perpetúa lo que se conoce como imperialismo académico, una visión poscolonial donde Occidente tiene el monopolio de los flujos de información científica. Como hemos comentado, expertos como Poynder plantean si el propio plan $\mathrm{S}$ es de hecho una posible solución o un impedimento al papel que pueda tener la América Latina respecto a su propia visión y promoción del acceso abierto (Poynder, 2019a, 2019b).

\section{Consideraciones finales}

Se debe destacar, en primer lugar, que el Plan S ha conseguido generar y dinamizar un interesante, completo e intenso debate sobre el acceso abierto a las publicaciones científicas, en el que se han recogido múltiples puntos de vista y opiniones. A raíz de ello, la segunda versión del plan ha dado respuesta a buena parte de las carencias y limitaciones que se habían manifestado por parte de la comunidad académica (sociedades científicas, sociedades académicas, editores, científicos). 
La versión actual constituye, en nuestra opinión, una apuesta decidida y un buen instrumento para acelerar la transición hacia el acceso abierto a la ciencia, aunque, evidentemente, habrá muchas cuestiones por pulir y mejorar. En este punto, suscribimos el conocido proverbio árabe "Quien quiere hacer algo encuentra un medio, quien no quiere hacer nada encuentra una excusa" y creemos que las propuestas son el mejor camino para transformar la realidad.

Por algunos de los aspectos que se han comentado, algunas incógnitas acechan sobre la implementación del Plan S. Aunque no se duda que los investigadores seguirán las pautas de la financiación (pasada la etapa del proselitismo inicial del acceso abierto, actualmente para incrementar el porcentaje de producción científica en abierto se ha optado por la obligación mediante políticas institucionales), está por ver el papel que van a tener las principales editoriales. Como se ha comentado, en muchas disciplinas, el modelo híbrido o el embargo de 12 meses eran una especie de guerra fría entre los intereses de los financiadores públicos y los intereses de las principales editoriales. Con el Plan S como jaque al modelo híbrido, habrá que ver si las principales revistas intentan mantenerse como vía de publicación de este 3-6\% de artículos o mantienen su propio modelo de negocio a la espera de que crezca este porcentaje de artículos. Evidentemente, el papel de países como Estados Unidos, la China, la India, pero también Alemania (y el estado español) con sus propias vías de desarrollo del acceso abierto pueden decantar la balanza en uno u otro sentido.

Lo que consideramos importante es que la presión y las dificultades de cumplimiento no se traspasen a investigadores, repositorios universitarios o bien a revistas científicas de editoriales universitarias, que a menudo asumen los costes de producción y que la vía diamante (publicación sin pago por el autor) no pueda seguir el ritmo de desarrollo técnico y requerimientos que las grandes editoriales van a poder sustentar de forma más fácil.

En estos momentos se ha empezado a mandar algunas señales claras del empuje del plan. El principal ejemplo lo encontramos en la convocatoria pública (tender) para proveer una plataforma de publicación en Europa, que contiene especificaciones que se ajustan a la "compliance" del Plan 5 y que servirá para lanzar un claro aviso a las editoriales y revistas de que la propuesta va en serio. Esta plataforma será sin duda una buena opción para los autores y, además, presiona a las revistas a cambiar.

Para finalizar, coincidimos con Lewis (2019) que el plan S va a tener un papel relevante para acelerar la disrupción en la comunicación científica, aunque habrá que ver, como apunta Kelly (2019), cuál va a ser la intensidad de sus efectos porque no es lo mismo un temblor que un terremoto.

\section{Notas al final}

1. https://www.coalition-s.org/

2.https://www.nature.com/articles/d41586-018-07659-5?- $f b c l i d=I$ w A R 0 i A A A V d Z L S F N N m J y 2 m 8 v r v Q16261TjdMRVMB5YOlyBSotYKhTVKAeh2MA

3. https://etendering.ted.europa.eu/cft/cft-display.html?cftld=5034

https://blogs.lse.ac.uk/impactofsocialsciences/2019/08/20/ fitting-the-mould-what-the-european-commissions-secondtender-for-an-open-research-publishing-platform-tells-usabout-the-future-of-scholarly-communication/

\section{Referencias}

AmeliCA (2019). Principios y valores. Latinoamérica: AmeliCA. Recuperado de http://amelica.org/index.php/principios-y-valores/

Amsterdam call for action on open science. (2016). Amsterdam: eu2016. Recuperado de https://www.government.nl/binaries/government/documents/reports/2016/04/04/amsterdam-call-for-actionon-open-science/amsterdam-call-for-action-on-open-science.pdf

Anglada, L., y Abadal, E. (2018). ¿Qué es la ciencia abierta? Anuario ThinkEPI, v. 12, pp. 292-298. https://doi.org/10.3145/thinkepi.2018.43

Becerril-García, A. (10 de enero de 2019). AmelicA vs Plan S: mismo objetivo, dos estrategias distintas para lograr el acceso abierto. Latinoamérica: Blog Amelica.org. Recuperado de http://amelica.org/ index.php/2019/01/10/amelica-vs-plan-s-mismo-objetivo-dos-estrategias-distintas-para-lograr-el-acceso-abierto/

Bernal, I. (2018). Plan S: Principios, hoja de ruta y reacciones. Enredadera (32). Recuperado de http://sitios.csic.es/web/enredadera/ plan_s

Bosman, J., y Kramer, B. (15 de junio de 2019). Nine routes towards Plan $S$ compliance - updated. Innovation in Scholarly Communication. Recuperado de https://101innovations.wordpress.com/tag/plan-s/

Burgman, M. (2019), Open access and academic imperialism. Conservation Biology, 33(1): 5-6. https://doi.org/10.1111/cobi.13248

cOAlition S. (2019). Feedback on the draft implementation guidance of Plan S [Dataset]. Zenodo. http://doi.org/10.5281/zenodo.3249905

de Bruin, B., Kamerlin, S. C. L., Copéret, C., Wittung-Stafshede, P., Derat, E., H., S., y van der Kamp, M. (2019). Researcher Response to the Revisions Made to the Plan S Principles and Implementation Guidance by cOAlition S (Version 1). Zenodo. https://doi.org/10.5281/ zenodo. 3243310

DORA (2012). San Francisco Declaration on Research Assessment. San Francisco: DORA. Recuperado de https://sfdora.org/read/

Eisen, M. (2018). Open Letter in Support of Funder Open Publishing Mandates. Berkeley: Michael Eisen. Recuperado de http:// michaeleisen.org/petition/

FOOA (2019). "The Fair Open Access Principles". coa. https://www. fairopenaccess.org/the-fair-open-access-principles/

Hicks, D., Wouters, P., Waltman, L., De Rijcke, S., y Rafols, I. (2015). The Leiden Manifesto for research metrics. Nature, 520(7548), 429-431. Recuperado de https://www.nature.com/news/bibliometrics-the-leiden-manifesto-for-research-metrics-1.17351

Kamerlin, L., et al. (2018). Reaction of Researchers to Plan S: Too Far, Too Risky. S.l.: Plan S Open Letter. Recuperado de https://sites.google. com/view/plansopenletter/open-letter

Kelly, E. (28 de febrero de 2019). The "Plan S" blog: Is the EU's open access plan a tremor or an earthquake?. Science Business. Recuperado de https://sciencebusiness.net/news/plan-s-blog-eus-openaccess-plan-tremor-or-earthquake

Kingsley, D. (1 de febrero de 2019). Plan S - links, commentary and 
news items. Unlocking Research: University of Cambridge Office for Scholarly Communication. Recuperado de https://unlockingresearch-blog.lib.cam.ac.uk/?p=2433

Lewis, D. W. (2019). Is Scholarly Publishing Like Rock and Roll?. IUPUI ScholarWorks Repository. Recuperado de http://hdl.handle. net/1805/20430

López-Borrull, A. (2019). 'Plan S': La velocidad del acceso abierto depende del punto de referencia?. Comeln. Revista dels Estudis de Ciències de la Informació i la Comunicació (84). Recuperado de https://www.uoc.edu/divulgacio/comein/es/numero84/articles/ plan-S-velocidad-acceso-abierto-punto-referencia.html

López-Borrull, A., Ollé-Castellà, C., Abadal, E., y García-Grimau, F. (en revisión). El Plan $S$ y el ecosistema de revistas españolas en Ciencias Sociales hacia el acceso abierto: amenazas y oportunidades

Martínez-Galindo, F. J., Rubio, F., Hernández-San-Miguel, J., y Fernández Burguete, S. (2019). Plan S: challenges and opportunities in Spain. Insights 32(1), 17. https://doi.org/10.1629/uksg.467

Pollock, D., y Michael, A. (24 de septiembre de 2018). News \& Views: potential impact of Plan S. Philadelphia: Delta Think. Recuperado de https://deltathink.com/news-views-potential-impact-of-plan-s/

Poynder, R., (15 de febrero de 2019a). Plan S: What strategy now for the Global South?. London: Richard Poynder. Recuperado de https:// richardpoynder.co.uk/Plan_S.pdf

Poynder, R., (21 de mayo de 2019b). The OA interviews: Arianna Becerril-García, Chair of AmeliCA. London: Open and shut?. Recuperado de https://poynder.blogspot.com/2019/05/the-oa-interviews-arianna-becerril.html

Quaderi, N., Hardcastle, J., Petrou, C., y Szomszoret, M. (2019). The Plan S footprint: Implications for the scholarly publishing landscape. Philadelphia, London:Institute for Scientific Information, Web of Science Group. Recuperado de https://clarivate.com/g/plan-s-footprint/

Sánchez-García, S. (12 de julio de 2019). ¿Estamos preparados para hacer frente a las exigencias del Plan S?. Aula Magna 2.0. Recuperado de https://cuedespyd.hypotheses.org/6417

Schiltz, M. (2018). Science Without Publication Paywalls: cOAlition $S$ for the Realisation of Full and Immediate Open Access. PLoS Med 15(9). https://doi.org/10.1371/journal.pmed.1002663

Schneider, L. (11 de junio de 2019). Researchers reject APC-based OA publishing as promoted by Plan S. For Better Science. Recuperado de https://forbetterscience.com/2019/06/11/researchers-reject-apcbased-oa-publishing-as-promoted-by-plan-s/

Schneider, L. (11 de septiembre de 2018). Response to Plan S from Academic Researchers: Unethical, Too Risky!. For Better Science. Recuperado de https://forbetterscience.com/2018/09/11/responseto-plan-s-from-academic-researchers-unethical-too-risky/

Villatoro, F. (4 de julio de 2019). "El descubrimiento científico usando minería de artículos científicos". La ciencia de la mula Francis. Recuperado de https://francis.naukas.com/2019/07/04/el-descubrimiento-cientifico-usando-mineria-de-articulos-cientificos/

Willighagen, E., Tennant, J. (2019). Open Science Feedback on the Guidance on the Implementation of Plan S. Zenodo. https://doi. org/10.5281/zenodo. 2560200

\section{CV}

Ernest Abadal. Catedrático de la Facultat de Biblioteconomia i Documentació de la Universitat de Barcelona. Director del Centro de Investigación en Información, Comunicación y Cultura. Dirige un proyecto de investigación sobre ciencia abierta en España. Es miembro del consejo asesor de diversas revistas científicas. https://fbd.ub.edu/pub/abadal

Alexandre López-Borrull. Profesor agregado de la Universitat Oberta de Catalunya, donde es director del Grado de Información y Documentación. Es doctor en Química por la Universitat Autònoma de Barcelona y Licenciado en Documentación por la UOC. Como investigador ha trabajado en aspectos legales de la información, las fuentes de información electrónica en ciencia y tecnología, y ha participado en diversos proyectos. Sus intereses de investigación están relacionados con el acceso abierto y la ciencia abierta. Forma parte del grupo de investigación Knowledge and Information Management in Organizations. https://www.directorioexit.info/ficha157

Candela Ollé Castellà. Profesora agregada de la Universitat Oberta de Catalunya, directora del Máster Universitario en Periodismo y Comunicación Digital: Datos y Nuevas Narrativas y Codirectora de la revista académica BiD: textos universitaris de Biblioteconomia i Documentació (UOC-UB). Licenciada en Periodismo por la Universitat Autònoma de Barcelona y Doctora en Documentación por la Universitat de Barcelona ha centrado su investigación en la comunicación científica, el acceso abierto y la ciencia abierta. Miembro del grupo de investigación Cultura i Continguts Digitals (UB) y colaboradora del grupo de investigación GAME (UOC). https://www.directorioexit.info/ficha1803

Francesc García Grimau. Profesor asociado del Departamento de Biblioteconomía, Documentación y Comunicación Audiovisual (UB) y consultor de bibliotecas en OCLC España. Es Graduado en Información y Documentación (UB) y estudiante del Máster Universitario en Gestión Estratégica de la Información y el Conocimiento en las Organizaciones (UOC). Su experiencia laboral se ha desarrollado en el ámbito de la documentación técnica en empresas industriales y en el de la consultoría especializada en software de gestión de la producción científica y de la investigación. https://www.directorioexit.info/ficha5340 